\title{
Trigger for group A streptococcal M1T1 invasive disease
}

\author{
Jason N. Cole,* Jason D. McArthur,* Fiona C. McKay,* Martina L. Sanderson-Smith,* \\ Amanda J. Cork, $*$ Marie Ranson, ${ }^{*}$ Manfred Rohde, ${ }^{\dagger}$ Andreas Itzek, ${ }^{\dagger}$ Hongmin Sun, ${ }^{\ddagger}$ \\ David Ginsburg, ${ }^{+,},, \|, \#$ Malak Kotb, ${ }^{* *}$ Victor Nizet, $^{\dagger \dagger}$ G. S. Chhatwal, ${ }^{\dagger}$ \\ and Mark J. Walker*,1 \\ *School of Biological Sciences, University of Wollongong, Wollongong, New South Wales, Australia; \\ ${ }^{\dagger}$ Department of Microbial Pathogenesis and Vaccine Development, German National Centre \\ for Biotechnology, Braunschweig, Germany; ${ }^{\ddagger}$ Life Sciences Institute, University of Michigan, \\ Ann Arbor, Michigan, USA; ${ }^{\S}$ Howard Hughes Medical Institute, ${ }^{\| D e p a r t m e n t ~ o f ~ I n t e r n a l ~ M e d i c i n e ~}$ \\ and ${ }^{\#}$ Department of Human Genetics, University of Michigan, Ann Arbor, Michigan, USA; \\ **Department of Molecular Science, University of Tennessee, Memphis, Tennessee, USA; and \\ ${ }^{\dagger \dagger}$ Department of Pediatrics, UCSD, La Jolla, California, USA
}

ABSTRACT The globally disseminated Streptococcus pyogenes M1T1 clone causes a number of highly invasive human diseases. The transition from local to systemic infection occurs by an unknown mechanism; however invasive M1T1 clinical isolates are known to express significantly less cysteine protease SpeB than M1T1 isolates from local infections. Here, we show that in comparison to the M1T1 strain 5448, the isogenic mutant $\Delta$ speB accumulated 75-fold more human plasmin activity on the bacterial surface following incubation in human plasma. Human plasminogen was an absolute requirement for M1T1 strain 5448 virulence following subcutaneous (s.c.) infection of humanized plasminogen transgenic mice. S. pyogenes M1T1 isolates from the blood of infected humanized plasminogen transgenic mice expressed reduced levels of SpeB in comparison with the parental 5448 used as inoculum. We propose that the human plasminogen system plays a critical role in group A streptococcal M1T1 systemic disease initiation. SpeB is required for $S$. pyogenes M1T1 survival at the site of local infection, however, SpeB also disrupts the interaction of $S$. pyogenes M1T1 with the human plasminogen activation system. Loss of SpeB activity in a subpopulation of $S$. pyogenes M1T1 at the site of infection results in accumulation of surface plasmin activity thus triggering systemic spread.-Cole, J. N., McArthur, J. D., McKay, F. C., Sanderson-Smith, M. L., Cork, A. J., Ranson, M., Rohde, M., Itzek, A., Sun, H., Ginsburg, D., Kotb, M., Nizet, V., Chhatwal, G. S., Walker, M. J. Trigger for group A streptococcal M1T1 invasive disease. FASEB J. 20, E1139-E1145 (2006)

Key Words: Streptococcus pyogenes $\cdot$ SpeB $\cdot$ plasminogen

EpIDemic Invasive group A Streptococcus (GAS, S. pyogenes) disease in the Western World since the mid-1980s has been paralleled by the emergence of a globally disseminated serotype M1T1 GAS clone (1). This M1T1 clone is associated with life-threatening infections such as necrotizing fasciitis and streptococcal toxic shock syndrome (2), while also causing uncomplicated infections in other patients (3). The mechanism that triggers the transition from local to invasive infection by GAS M1T1 is unknown, however, epidemiologic studies have documented an inverse relationship between human invasive disease and the expression of the streptococcal pyrogenic exotoxin B (SpeB) (4). SpeB is a secreted cysteine protease initially expressed as a 40 $\mathrm{kDa}$ zymogen, which is then converted to its $28 \mathrm{kDa}$ active form by autocatalytic truncation (5). SpeB is known to cleave host proteins including extracellular matrix (ECM) components, cytokine precursors, immunoglobulins, and antimicrobial peptides $(1,6,7)$ and has been shown to play an important role in establishing localized skin infections (8). SpeB also degrades GAS virulence factors including the cell wallassociated fibrinogen-binding antiphagocytic M1 protein $(9,10)$, various superantigens $(11,12)$, and the secreted plasminogen activator streptokinase (13).

The capacity of GAS to assemble on its surface a trimolecular complex containing streptokinase, fibrinogen, and plasminogen has been correlated with a propensity for invasive infection (14). GAS is a highly specific human pathogen and the streptokinase produced by this organism displays a much greater affinity for human plasminogen than mouse plasminogen (15). A major advance in the understanding of GAS pathogenesis was attained by Sun et al. (15) who established a humanized plasminogen transgenic mouse model. These researchers demonstrated that streptokinase and the activation of plasminogen to plasmin is critical for GAS dissemination in vivo, leading to the proposal that human plasminogen

\footnotetext{
${ }^{1}$ Correspondence: School of Biological Sciences, University of Wollongong, Wollongong, NSW, 2522, Australia. Email: mwalker@uow.edu.au

doi: 10.1096/fj.06-5804fje
} 
is hijacked for use as a virulence factor by GAS $(15,16)$. In this study, we utilize the representative wild-type (WT) clonal M1T1 strain 5448 and the precise, in-frame allelic replacement mutant $\Delta s p e B(11,17)$ to investigate the interplay between $\mathrm{SpeB}$ and human plasminogen coopted to the bacterial surface.

\section{MATERIALS AND METHODS}

\section{Strains, media, and culture conditions}

S. pyogenes strain 5448 (M1T1) and the isogenic $\Delta s p e B$ mutant have been described previously (12). All $S$. pyogenes strains were routinely cultured at $37^{\circ} \mathrm{C}$ on horse-blood agar (BioMérieux, Sydney, NSW, Australia) or in static liquid cultures of Todd-Hewitt broth (Difco, Sydney, NSW, Australia) supplemented with $1 \%(\mathrm{w} / \mathrm{v})$ yeast extract (THBY medium). When required, SpeB was inactivated by growth in the presence of cysteine protease inhibitor E64 (N-[N-(L-3-transcarboxyirane-2-carbonyl)-L-Leucyl]-agmatine) (Sigma, Sydney, NSW, Australia) at a final concentration of $28 \mu \mathrm{M}$.

\section{Hyaluronic acid capsule determination}

Overnight cultures of $S$. pyogenes were diluted 1:10 in fresh THBY media and grown to an optical density (OD) at $600 \mathrm{~nm}$ $\left(\mathrm{OD}_{600}\right)$ of $0.5-0.6$. The hyaluronic acid capsule was extracted and quantified by the stains-all method (18).

\section{Preparation of culture supernatant proteins}

An overnight culture of $S$. pyogenes was diluted 1:10 in fresh THBY medium and incubated at $37^{\circ} \mathrm{C}$ to late stationary phase (approx. $16 \mathrm{~h}$ ). The culture supernatant was harvested by centrifugation at $8000 \mathrm{~g}$ for $20 \mathrm{~min}$ at $4^{\circ} \mathrm{C}$ and filter sterilized through a $0.22 \mu \mathrm{m}$ filter (Millipore, Sydney, NSW, Australia) for use in SpeB activity assays. For sodium dodecyl sulfatepolyacrylamide gel electrophoresis (SDS-PAGE) analysis, equal volumes of cell-free supernatant and $10 \%(\mathrm{v} / \mathrm{v})$ trichloroacetic acid were mixed and incubated on ice for $1 \mathrm{~h}$. Following centrifugation at $15,000 \mathrm{~g}$ for $20 \mathrm{~min}$ at $4^{\circ} \mathrm{C}$, the pellet was washed with ice-cold $100 \%$ ethanol and centrifuged at $15,000 \mathrm{~g}$ for $15 \mathrm{~min}$ at $4^{\circ} \mathrm{C}$. The ethanol was removed by aspiration, the pellet air-dried for $30 \mathrm{~min}$ and resuspended in $1 \mathrm{ml}$ of $100 \mathrm{mM}$ Tris-HCl ( $\mathrm{pH}$ 7.6).

\section{SpeB activity assays}

Cysteine protease SpeB activity in S. pyogenes culture supernatants was determined according to the method of Hytönen et al. (19). To examine SpeB activity of large numbers of $S$. pyogenes colonies, the Columbia skim milk plate-based assay was used according to the method of Ashbaugh et al. (20).

\section{Immunogold electron microscopy}

S. pyogenes were fixed with $0.2 \%$ glutaraldehyde and $0.5 \%$ formaldehyde for $1 \mathrm{~h}$ on ice, washed with cacodylate buffer (0.1 M cacodylate, $0.9 \mathrm{M}$ sucrose, $0.01 \mathrm{M} \mathrm{MgCl}_{2}, 0.01 \mathrm{M}$ $\mathrm{CaCl}_{2} ; \mathrm{pH}$ 6.9) containing $10 \mathrm{mM}$ glycine and dehydrated with ethanol following the progressive lowering of temperature protocol $(10 \%, 30 \%$ ethanol on ice; $50 \%$ ethanol at $-20^{\circ} \mathrm{C} ; 70 \%, 90 \%, 100 \%$ ethanol at $-30^{\circ} \mathrm{C}$; each step for 30 min). Samples were infiltrated with the Lowicryl ${ }^{\circledR}$ resin K4M over $3 \mathrm{~d}$ before polymerization at $-30^{\circ} \mathrm{C}$ with $\mathrm{UV}$ light (366 $\mathrm{nm}$ ) for $2 \mathrm{~d}$ and $1 \mathrm{~d}$ at room temperature. Ultrathin sections were collected with formvar-coated cover-slips and incubated with SpeB specific IgG antibodies $\left(75 \mu \mathrm{g} / \mathrm{ml}, 10 \mathrm{~h}\right.$ at $\left.4^{\circ} \mathrm{C}\right)$, washed with $\mathrm{PBS}$ and bound antibodies were made visible with protein A/G coated gold-particles (10 or $15 \mathrm{~nm}$ in size). Samples were counterstained with uranyl acetate for $1 \mathrm{~min}$ and air-dried before examination in a Zeiss transmission electron microscope EM910 at an acceleration voltage of $80 \mathrm{kV}$.

\section{Plasminogen binding and cell surface plasmin activity}

The binding of ${ }^{125}$ I-plasminogen was measured as reported elsewhere (14). Approximately $70 \mathrm{ng}$ of ${ }^{125}$ I-plasminogen was added to $250 \mu \mathrm{l}$ of cell suspension and incubated at room temperature for $45 \mathrm{~min}$. The cells were harvested by centrifugation, the supernatant carefully aspirated and pellet-associated radioactivity measured using an automatid Wallac gamma counter (Perkin Elmer, Jugesheim, Germany). The results were expressed as a percentage of input radioactivity. All measurements were determined in triplicate.

Plasmin acquisition in human plasma by $S$. pyogenes isolates was determined essentially as described previously (21). Frozen plasma was purchased from the Red Cross Blood Bank (Sydney, NSW, Australia), defrosted on ice and pooled. Aliquots of pooled plasma were depleted of plasminogen by incubation at $4^{\circ} \mathrm{C}$ on ice with excess lysine-sepharose ${ }^{\circledR} 4 \mathrm{~B}$ for 1-2 $\mathrm{h}$ with gentle agitation. The extent of plasminogen depletion and the plasmin activity of plasma was determined by chromogenic assay with Spectrozyme ${ }^{\circledR}$ PL (American Diagnostics Inc., Stamford, CT, USA), in the presence and absence of streptokinase, and by Western blot using a polyclonal rabbit anti-human plasminogen antibody (Calbiochem, Kilsyth, VIC, Australia).

GAS were cultured overnight as stationary cultures in $25 \mathrm{ml}$ THY inoculated with 1 colony. GAS were pelleted by centrifugation at $800 \mathrm{~g}$, washed in $50 \mathrm{ml}$ of PBS, pH 7.4, prewarmed to $37^{\circ} \mathrm{C}$, and resuspended to $\mathrm{OD}_{600}=0.7$ (corresponding to $\log$ phase, in which streptokinase secretion is induced). A 2 $\mathrm{ml}$ aliquot of this suspension was pelleted as above and resuspended in an equal vol of $100 \%$ plasma or plasminogendepleted plasma at $37^{\circ} \mathrm{C}$. GAS were incubated in plasma for $3 \mathrm{~h}$ at $37^{\circ} \mathrm{C}$, pelleted by centrifugation and washed twice with 1 vol of ice-cold $0.01 \mathrm{M}$ EDTA, $0.1 \%(\mathrm{w} / \mathrm{v})$ gelatin in PBS, $\mathrm{pH}$ 7.4. GAS were resuspended in $0.1 \%(\mathrm{w} / \mathrm{v})$ gelatin in PBS, $\mathrm{pH}$ 7.4 to $\mathrm{OD}_{600}=0.75$. Aliquots $(100 \mu \mathrm{l})$ of this suspension were incubated in triplicate in the presence and absence of $20 \mu \mathrm{l}$ Spectrozyme ${ }^{\circledR} \mathrm{PL}, 2.5 \mathrm{mM}$ at $37^{\circ} \mathrm{C}$ for $60 \mathrm{~min}$ in a 96 -well plate. The reaction was quenched with $80 \mu \mathrm{l}$ of $1.75 \mathrm{M}$ acetic acid, the plates centrifuged at $800 \mathrm{~g}$, and $\mathrm{A}_{405}$ of supernatants determined.

Plasmin activity was determined as the difference between $\mathrm{A}_{405}$ in the presence and absence of substrate, thus accounting for differences in the sedimentation efficiency of GAS isolates. Each isolate was assayed in at least two independent experiments. Plasmin equivalents and the linear range of the assay $\left(\mathrm{A}_{405}=0-0.6\right)$ were determined using a standard curve of purified plasmin (Roche Diagnostics, GmbH, Mannheim, Germany).

\section{Extraction of cell wall-associated proteins}

Mutanolysin cell wall extracts were prepared from stationary phase cultures of $S$. pyogenes as reported elsewhere (22).

\section{Immunological reagents}

Affinity-purified rabbit anti-SpeB IgG was purchased from Toxin Technology Inc., Sarasota, FL, USA. Polyclonal rabbit 
antibodies were raised against purified recombinant $S$. pyogenes streptococcal enolase (SEN) and glyceraldehyde-3-phosphate dehydrogenase (GAPDH). A rabbit polyclonal antiserum was raised against Lancefield group C streptokinase (Sigma), which shares $>90 \%$ identity with the streptokinase (Ska) protein of $S$. pyogenes. All rabbit immunizations were performed according to the method of Gillen et al. (23). The production of mouse polyclonal antiserum against M1 protein is described elsewhere (24).

\section{Western blot analysis}

SDS-PAGE was performed according to the method of Laemmli (25). Cell wall extracts or culture supernatant proteins were transferred to nitrocellulose membrane at 100 $\mathrm{V}$ for $1 \mathrm{~h}$ using the Mini Trans-Blot ${ }^{\circledR}$ (Bio-Rad) (26). The membranes were blocked in a solution of $5 \%(\mathrm{w} / \mathrm{v})$ skim milk (Difco) in PBS (137 mM NaCl, $2.7 \mathrm{mM} \mathrm{KCl,} 7.9 \mathrm{mM}$ $\mathrm{Na}_{2} \mathrm{HPO}_{4}, 1.5 \mathrm{mM} \mathrm{KH} \mathrm{PO}_{4} ; \mathrm{pH} 7.4$ ) for $1 \mathrm{~h}$ at room temperature. After a $10 \mathrm{~min}$ wash with PBS, the membranes were incubated for $2 \mathrm{~h}$ with primary Ab diluted 1:1,000 in PBS. Following three washes for $10 \mathrm{~min}$ each with PBS, the membranes were incubated for $1 \mathrm{~h}$ with a 1:1,000 dilution of goat anti-rabbit IgG HRP conjugate (Bio-Rad) for SpeB, Ska, SEN, and GAPDH blots, or a 1:1,000 dilution of goat antimouse IgG HRP conjugate (Bio-Rad) for M1 protein blots. Excess secondary Ab was removed by three PBS washes for 10 min each and all blots developed in a solution of $100 \mathrm{mM}$ Tris- $\mathrm{HCl}$ ( $\mathrm{pH} 7.6$ ) containing $1.4 \mathrm{mM}$ diaminobenzidine and $0.06 \%(\mathrm{v} / \mathrm{v})$ hydrogen peroxide.

\section{Protease assays}

SpeB protease ( $5 \mu \mathrm{g}$; Toxin Technology Inc.) was mixed with $5 \mu \mathrm{g}$ of purified human plasminogen or fibrinogen (14), adjusted to final vol of $25 \mu \mathrm{l}$ with PBS ( $\mathrm{pH} 7.4$ ) and incubated at $37^{\circ} \mathrm{C}$ for $3 \mathrm{~h}$. Cleavage of substrate protein was determined by SDS-PAGE analysis. Positive controls containing $5 \mu \mathrm{g}$ SpeB and $25 \mu \mathrm{g} \beta$-casein (Sigma) were used in all assays. Negative controls containing protease or substrate only were also included.

\section{Streptococcal infection model}

AlbPLG1 transgenic mice $\left(\mathrm{Tg}^{+}\right)$(27) heterozygous for the human plasminogen transgene were backcrossed greater than $n=6$ with C57BL/J6 mice (Animal Resources Centre, Perth, Australia). Nontransgenic $\left(\mathrm{Tg}^{-}\right)$littermates were used as controls in all experiments. Streptococcal strains used for inocula were harvested at midlog phase $\left(\mathrm{OD}_{600}\right.$ approx. 0.5). Bacteria were washed with an equal vol of sterile $0.7 \%$ saline and diluted to the required inoculum. The number of viable bacteria present was determined by counting colony-forming units (CFU) after plating a dilution series onto blood agar plates (BioMérieux). Groups of $10 \mathrm{Tg}^{+}$mice and $\mathrm{Tg}^{-}$littermates were infected with either $2.8 \times 10^{8} \mathrm{CFU}$ (5448) or $1.35 \times 10^{8} \mathrm{CFU}(\Delta s p e B$ mutant $)$ via a s.c. injection into a shaved region of the right flank of each mouse. Mortality was recorded over $14 \mathrm{~d}$.

For experiments examining the dissemination of streptococci during infection, a separate cohort of $\mathrm{Tg}^{+}$mice was inoculated s.c. Wild-type 5448 was inoculated using $1.58 \times$ $10^{9} \mathrm{CFU}(n=4 ; 1$ mouse succumbed to infection on day 2 ) and $1.49 \times 10^{8} \mathrm{CFU}(n=1)$. The 5448 mutant $\Delta$ speB was inoculated using $1.71 \times 10^{9} \mathrm{CFU}$ (mutant; $n=4 ; 1$ mouse succumbed to infection on day 2) and $4.5 \times 10^{7} \mathrm{CFU}(n=1)$. On day 3 postinfection, mice were sacrificed by $\mathrm{CO}_{2}$ asphyxiation, and representative bacteria were isolated from the site of initial infection and bacterial loads present in the bloodstream were determined by plating serial dilutions onto blood agar. All experiments were conducted according to the Guidelines for the Care and Use of Laboratory Animals (National Health and Medical Research Council, Australia) and were approved by the University of Wollongong Animal Ethics Committee.

\section{Statistical analyses}

Average capsular hyaluronic acid, SpeB activity and plasminogen binding for 5448 and $\Delta s p e B$ were compared using the two-tailed unpaired $t$ test. Cell surface plasmin activity levels were evaluated using the Welch ANOVA (assuming unequal variances). Differences in survival of $\mathrm{Tg}^{+}$and $\mathrm{Tg}^{-}$mice infected with 5448 and $\Delta s p e B$ were determined by the log- a

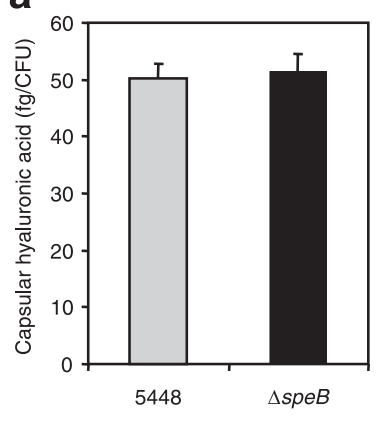

C

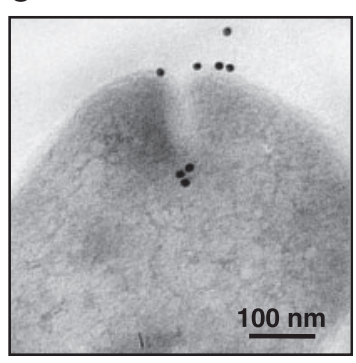

d

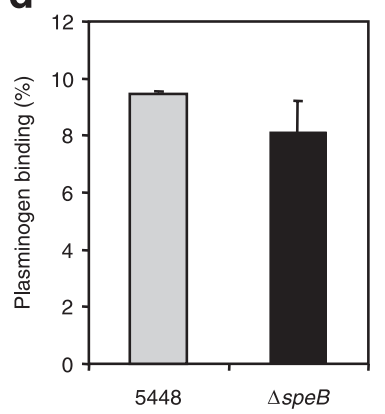

e

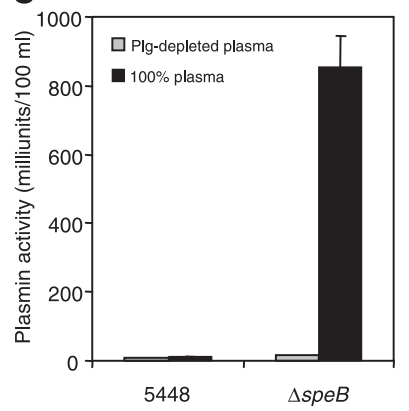

Figure 1. In vitro characterization of M1T1 S. pyogenes strain 5448 (WT) and the isogenic $\Delta$ speB mutant. a) 5448 and the $\Delta$ speB mutant express equivalent amounts of capsular hyaluronic acid (mean \pm SD). b) Secreted SpeB protease activity (mean $\pm \mathrm{SD}$ ) is abolished in the $\Delta s p e B$ mutant compared with 5448. c) Electron microscopic analyses detected SpeB zymogen at the ExPortal microdomain of 5448, but not $\Delta$ speB (data not shown). d) Equivalent amounts of purified human ${ }^{125} \mathrm{I}$ labeled plasminogen (mean \pm SD) are bound by 5448 and $\Delta s p e B$. e) The $\Delta s p e B$ mutant cell surface accrues a 75 -fold higher concentration of human plasma activity (mean \pm SD) compared with strain 5448 . 
rank test. Mean bacterial loads in the bloodstream $\left(\log _{10}\right.$ CFU) of $\mathrm{Tg}^{+}$mice infected with 5448 or $\Delta s p e B$ were compared using the two-tailed unpaired $t$ test. The $t$ and log-rank tests were performed using GraphPad Prism version 4.02 (GraphPad Software Inc., San Diego, CA, USA). The Welch ANOVA was performed using JMP (SAS Institute, NC, USA).

\section{RESULTS}

\section{Characterization of M1T1 strains 5448 and $\Delta s p e B$}

Both WT and isogenic $\Delta s p e B$ mutant strains expressed identical amounts of hyaluronic acid capsule (Fig. 1a), excluding a pleiotropic mutation that confounded earlier studies of SpeB in GAS pathogenesis (20). Secreted cysteine protease activity was undetectable in the $\Delta s p e B$ mutant $(P \leq 0.05$; Fig. $1 b)$, and SpeB zymogen was detected only at the cell wall ExPortal microdomain (28) of the parent M1T1 strain (Fig. 1c). Nonetheless, both WT and $\triangle s p e B$ mutant bound equivalent amounts of purified human plasminogen (Fig. $1 d$ ). These data indicate that, like the GAS M5 strain characterized by Rosch and Caparon (28), the M1T1 clone also secretes SpeB zymogen via the ExPortal microdomain. The presence or absence of SpeB does not affect the capacity of M1T1 GAS to bind purified human plasminogen, suggesting that the surface plasminogen receptors $\alpha$-enolase (SEN) (29) and GAPDH (30) are not disabled by SpeB proteolysis. However, in comparison to the WT strain, the isogenic $\Delta s p e B$ mutant accumulated 75-fold higher levels of human plasmin activity on the bacterial surface following incubation in human plasma for $3 \mathrm{~h}$ at $37^{\circ} \mathrm{C}(P \leq 0.05$; Fig. $1 e)$.

\section{Analysis of plasminogen activation system components}

To elucidate the underlying reason(s) for the enhanced capacity of the cysteine protease-deficient GAS strain to accumulate surface plasmin activity, we compared WT and $\Delta$ speB mutant strains for the expression of cell wall-associated plasminogen and fibrinogen receptors and for secretion of streptokinase. Active 28 $\mathrm{kDa}$ SpeB was identified only in supernatants of the WT strain; with addition of the SpeB inhibitor E64 just the $40 \mathrm{kDa}$ SpeB zymogen form was detected (Fig. 2a). Streptokinase was present only in supernatants of the $\Delta$ speB mutant or the WT parent strain grown in the presence of E64 (Fig. 2a). The fibrinogen-binding M1 protein was found in cell wall extracts only in the absence of SpeB activity, whereas plasminogen receptors SEN and GAPDH were detected in cell wall extracts of both WT and $\Delta$ speB mutant (Fig. $2 a$ ). SpeB was also observed to degrade plasminogen directly, as well as fibrinogen, which forms a trimolecular complex with streptokinase and plasminogen bound to the GAS surface $(14,31)$ (Fig. 2b). Thus, SpeB interferes with the accumulation of GAS surface bound plasmin activity by cleaving four critical factors: plasminogen, the plasminogen activator streptokinase, fibrinogen and the fibrinogen-binding M1 protein (16).

\section{Infection of humanized plasminogen transgenic mice}

We next took advantage of heterozygous AlbPLG1 mice that express a human plasminogen transgene $\left(\mathrm{Tg}^{+}\right)$ (15) and littermate C57BL/J6 control mice $\left(\mathrm{Tg}^{-}\right)$to examine the virulence of WT M1T1 GAS and the

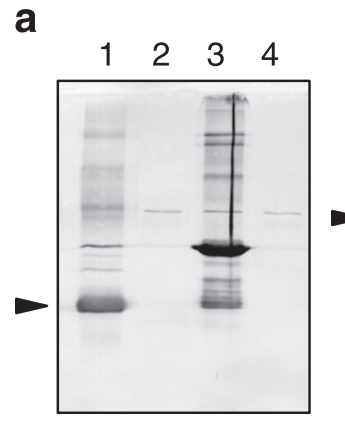

anti-SpeB

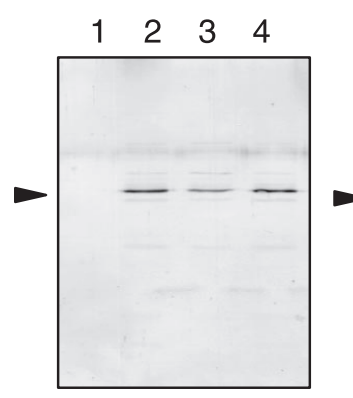

anti-Ska

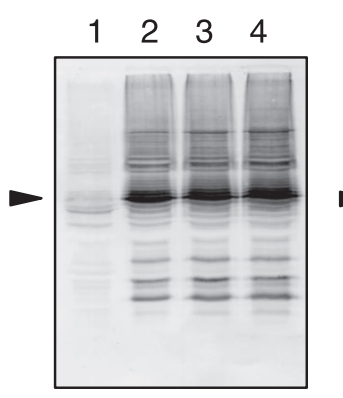

anti-M1

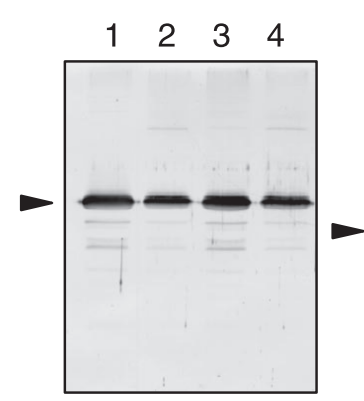

anti-SEN

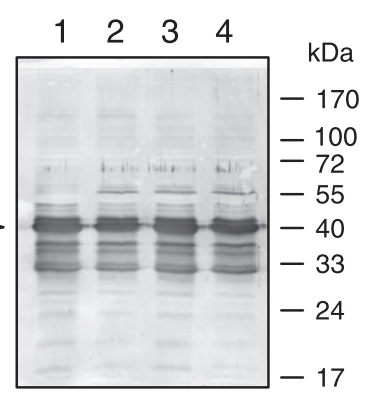

anti-GAPDH b

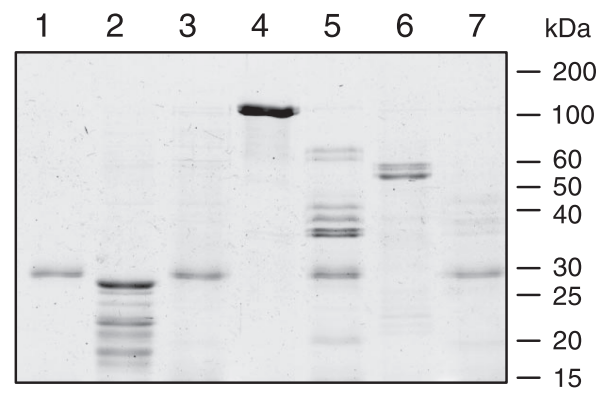

Figure 2. Western blot analysis and in vitro SpeB protease assays. a) Blots of secreted (SpeB and Ska) or cell wall-associated proteins (M1, $\mathrm{SEN}$, and GAPDH) harvested in the presence or absence of SpeB inhibitor E64 for 5448 (WT) and the isogenic $\Delta s p e B$ mutant. Lane 1, 5448 culture supernatant or cell wall (-E64); lane 2, $\Delta$ speB culture supernatant or cell wall (-E64); lane 3, 5448 culture supernatant or cell wall $(+\mathrm{E} 64)$; lane $4, \Delta s p e B$ culture supernatant or cell wall $(+\mathrm{E} 64)$. Filled arrowheads indicate major immunoreactive bands. $b$ ) SpeB protease degrades human plasminogen and fibrinogen, which play a key role in the accumulation of surface-bound plasmin activity by $S$. pyogenes. Lane 1, SpeB; lane $2, \beta$-casein; lane 3, SpeB and $\beta$-casein (positive control); lane 4, plasminogen; lane 5, SpeB and plasminogen; lane 6, fibrinogen; lane 7, SpeB and fibrinogen. Molecular mass markers are given in kilo-Daltons (kDa). 
a

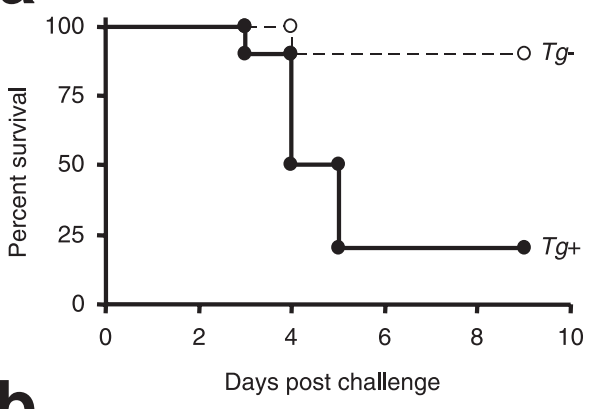

b

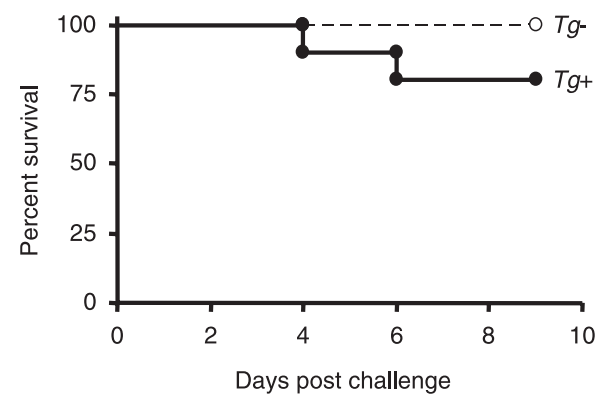

C
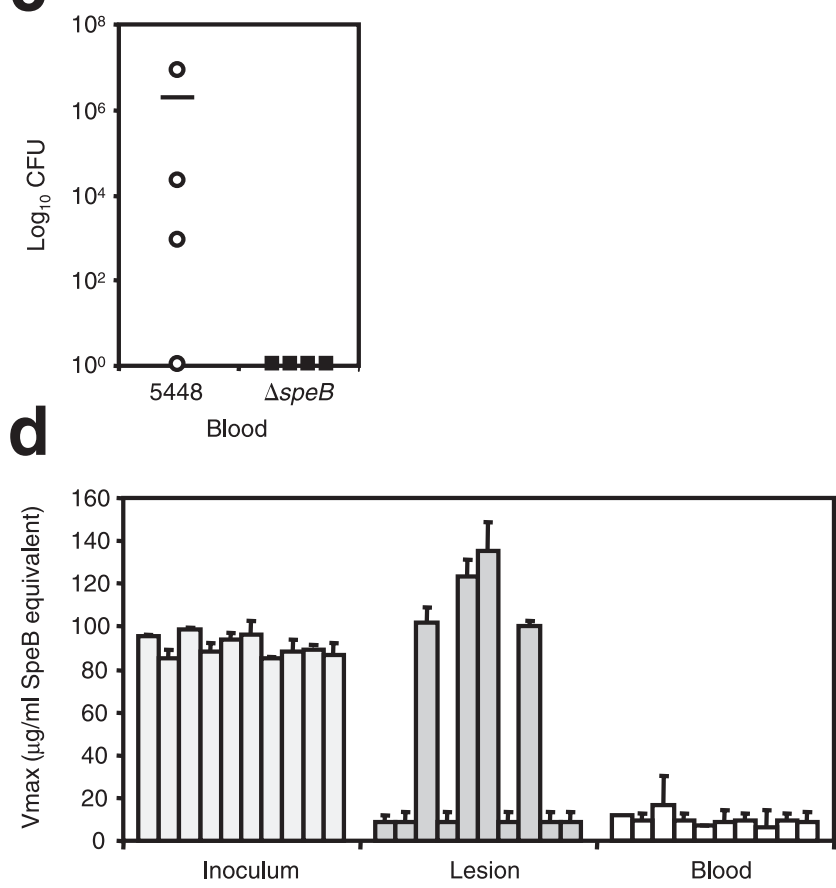

Figure 3. Survival curves, bacterial counts, and SpeB expression phenotype following s.c. infection of humanized plasminogen transgenic mice $\left(T g^{+}\right)$and nontransgenic control mice $\left(\mathrm{Tg}^{-}\right)$with $S$. pyogenes strain 5448 (WT) or the isogenic $\Delta s p e B$ mutant. a) The virulence of 5448 is increased in transgenic mice compared with the nontransgenic control. $b$ ) The virulence of the $\Delta s p e B$ mutant was attenuated in both transgenic and control mice. c) Bacterial counts in the bloodstream of $\mathrm{Tg}^{+}$mice $72 \mathrm{~h}$ postinfection are significantly higher for 5448 compared with $\Delta$ speB. d) Compared with the SpeB activity of 5448 colonies isolated from the inoculum, the $5448 \mathrm{Tg}^{+}$lesion isolates exhibit a varied SpeB-expression phenotype, while the $5448 \mathrm{Tg}^{+}$blood isolates have significantly reduced SpeB activity.

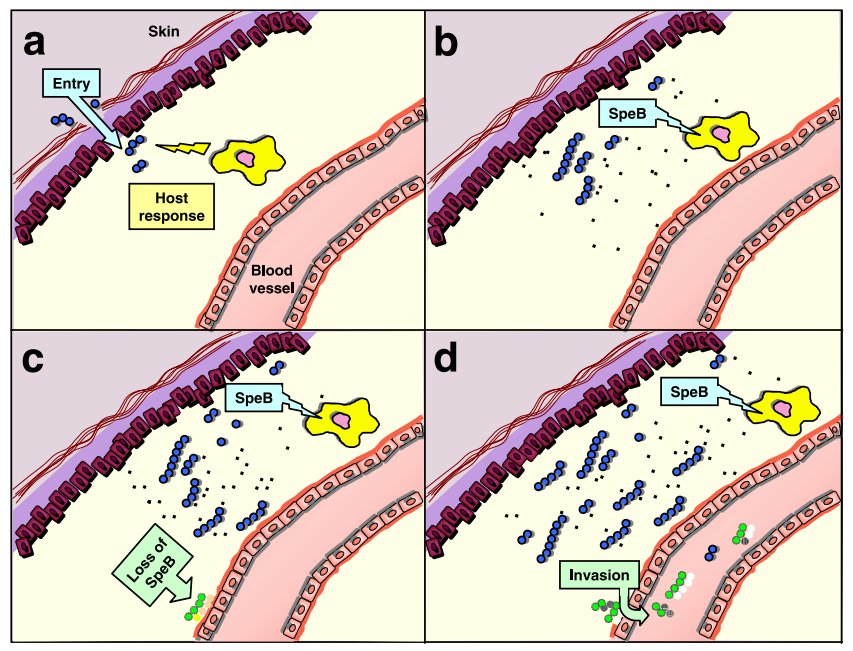

Figure 4. Proposed model for group A streptococcal systemic disease initiation. a) S. pyogenes M1T1 (blue) gain entry through the skin and a host innate immune response is initiated. b) During the initial stages of infection, S. pyogenes M1T1 express SpeB to combat the host response. c) Loss of SpeB activity in a subpopulation of group A streptococci (green) leads to the accumulation of surface plasmin activity. d) Transition of $S$. pyogenes M1T1 is facilitated by surface plasmin activity, resulting in systemic infection.

isogenic $\Delta s p e B$ mutant in a s.c. infection model. Only through use of this transgenic mouse line can one investigate the interaction of GAS with the plasminogen activation system, since this interaction is highly specific for human plasminogen $(15,16)$. In comparison to infection of nontransgenic control mice, the virulence of WT M1T1 strain was significantly increased in $\mathrm{Tg}^{+}$mice $(P \leq 0.05 ; 10 \%$ vs. $80 \%$ mortality) (Fig. $3 \boldsymbol{a}$ ). This result indicates that the human plasminogen system plays a crucial role in the ability of M1T1 GAS to initiate a lethal systemic infection. In comparison to the WT GAS parent strain, the isogenic $\Delta s p e B$ mutant was attenuated for virulence in the $\mathrm{Tg}^{+}$plasminogen-humanized mice $(P \leq 0.05 ; 80 \%$ vs. $20 \%$ mortality) (Fig. $3 a, b)$. Additionally, in comparison to GAS strain 5448, $72 \mathrm{~h}$ postinfection of $\mathrm{Tg}^{+}$mice the isogenic $\Delta s p e B$ mutant displayed significantly fewer bacterial counts in the blood ( $P \leq 0.05$; Fig. $3 c)$. These data support a role for SpeB in GAS survival at the site of local infection in the skin, as proposed by Svensson et al. (8), and suggested this difference may translate into an overall decreased lethality on challenge with the $\Delta s p e B$ mutant.

To further examine the interaction between the human plasminogen system and GAS cysteine protease SpeB, we examined the SpeB expression status of WT GAS isolated from the blood of the infected plasminogen-humanized $\mathrm{Tg}^{+}$mice. The WT GAS used in the initial challenge uniformly expressed high levels of cysteine protease activity ( $100 \%$ of inoculum colonies tested; $n=100$ ). Bacteria isolated from the s.c. site of infection expressed a mixed phenotype $(74 \%, 66 \%$, $63 \%$, and $31 \%$ of lesion recovered colonies SpeBnegative from 4 s.c. inoculated $\mathrm{Tg}^{+}$mice). Bacteria recovered from the blood unvaryingly demonstrated 
very low SpeB activity ( $100 \%$ of blood isolated colonies examined SpeB-negative) (Fig. $3 d$ ). This finding is consistent with the epidemiologic observation that M1T1 clinical isolates from human invasive disease express reduced levels of SpeB (4).

\section{DISCUSSION}

The mechanism utilized by GAS to switch from localized to systemic infection is presently unknown. However, subversion of the host plasminogen activation system is thought to play a key role $(15,16)$. In this study, we have shown that the absence of cysteine protease SpeB activity is required for the accumulation of plasmin activity on the cell surface of M1T1 GAS. In a humanized plasminogen transgenic mouse model, the loss of SpeB activity at the site of infection triggers the systemic dissemination of M1T1 GAS in vivo. These data support the proposal that human plasminogen plays a critical role in the initiation of GAS invasive disease.

Plasminogen is the proenzyme form of plasmin, a blood clot-dissolving serine protease, which degrades ECM components and activates matrix metalloproteases (32). In $S$. pyogenes, two distinct pathways have been identified for the binding of plasminogen to the cell surface. The direct pathway is mediated by the plasminogen-binding GAS M-like protein (PAM) (33), streptococcal enolase (SEN) (29), and GAPDH (29, $34)$. Indirect plasminogen-binding requires the formation of a trimolecular complex between plasminogen, streptokinase, and fibrinogen, which is bound to the GAS cell surface via plasminogen or fibrinogen receptors $(14,31,35)$. Human plasminogen is converted to plasmin by the plasminogen activators urokinase (uPA) and tissue-type plasminogen activator (tPA), or by secreted microbial activators including the GAS virulence factor streptokinase (36). Here, we demonstrate that the presence of SpeB does not interfere with the binding of plasminogen to the GAS cell surface. However, SpeB degrades four key factors required for the accumulation of surface plasmin activity on the GAS surface: plasminogen, streptokinase, fibrinogen, and the fibrinogen-binding M1 protein.

Utilizing a speB-negative mutant and a humanized in vivo model for impetigo in which neonatal foreskin was engrafted onto the hind flanks of CB-17 scid mice, Svensson et al. (8) determined that SpeB protease plays a critical role in the establishment of host tissue tropism by GAS. Our accumulated data suggest that SpeB, while contributing to localized infection, simultaneously mitigates the potential interaction of M1T1 GAS with the human plasminogen activation system. The ablation of SpeB expression in WT M1T1 GAS in the blood of plasminogen-humanized $\mathrm{Tg}^{+}$mice indicates that the loss of SpeB activity in a subpopulation of bacteria occurs at the site of infection, allowing accumulation of surface plasmin activity. This subpopulation thus gain enhanced invasive propensity, favoring transition of
GAS from the site of infection to the blood. In our model, vascular leakage induced by M protein complexing with fibrinogen to activate heparin binding protein release from neutrophils, may provide a source of plasminogen at the site of infection (37) (Fig. 4). The subpopulation of GAS undergoing a phase shift to eliminate SpeB expression gain not only the capacity to accumulate cell surface plasmin activity and spread systemically, but also elaborate greater levels of superantigens (12), the causative agents of streptococcal toxic shock.

The expression of the streptococcal cysteine protease SpeB and the human invasive disease severity are inversely related in M1T1 clonal isolates (4). The model we propose for GAS human systemic disease initiation accounts for this previously perplexing clinical observation and describes a mechanism by which systemic disease initiation occurs. The elucidation of the mechanism by which GAS causes human invasive disease will inform future treatment and prevention strategies.

We thank K. S. Sriprakash (Queensland Institute of Medical Research, Queensland, Australia) for providing M1 protein antiserum. J. N. Cole, M. L. Sanderson-Smith, and A. J. Cork are the recipients of an Australian Postgraduate Award. D. Ginsburg is a Howard Hughes Medical Institute Investigator. This work was supported by the National Health and Medical Research Council of Australia and a National Institute of Health grant (PO1HL057346).

\section{REFERENCES}

1. Cunningham, M. W. (2000) Pathogenesis of group A streptococcal infections. Clin. Microbiol. Rev. 13, 470-511

2. Stevens, D. L. (1992) Invasive group A Streptococcus infections. Clin. Infect. Dis. 14, 2-11

3. Johnson, D. R., Wotton, J. T., Shet, A., and Kaplan, E. L. (2002) A comparison of group A streptococci from invasive and uncomplicated infections: Are virulent clones responsible for serious streptococcal infections? I. Infect. Dis. 185, 1586-1595

4. Kansal, R. G., McGeer, A., Low, D. E., Norrby-Teglund, A., and Kotb, M. (2000) Inverse relation between disease severity and expression of the streptococcal cysteine protease, SpeB, among clonal M1T1 isolates recovered from invasive group A streptococcal infection cases. Infect. Immun. 68, 6362-6369

5. Musser, J., Stockbauer, K., Kapur, V., and Rudgers, G. (1996) Substitution of cysteine 192 in a highly conserved Streptococcus pyogenes extracellular cysteine protease (interleukin $1 \beta$ convertase) alters proteolytic activity and ablates zymogen processing. Infect. Immun. 64, 1913-1917

6. Hynes, W. (2004) Virulence factors of the group A streptococci and genes that regulate their expression. Front. Biosci. 9, 33993433

7. Nyberg, P., Rasmussen, M., and Björck, L. (2004) $\alpha_{2}$-Macroglobulin-proteinase complexes protect Streptococcus pyogenes from killing by the antimicrobial peptide LL-37. J. Biol. Chem. 279, $52820-52823$

8. Svensson, M. D., Scaramuzzino, D. A., Sjöbring, U., Olsen, A., Frank, C., and Bessen, D. E. (2000) Role for a secreted cysteine proteinase in the establishment of host tissue tropism by group A streptococci. Mol. Microbiol. 38, 242-253

9. Ringdahl, U., Svensson, H. G., Kotarsky, H., Gustafsson, M., Weineisen, M., and Sjöbring, U. (2000) A role for the fibrinogen-binding regions of streptococcal $\mathrm{M}$ proteins in phagocytosis resistance. Mol. Microbiol. 37, 1318-1326

10. Raeder, R., Woischnik, M., Podbielski, A., and Boyle, M. D. (1998) A secreted streptococcal cysteine protease can cleave a 
surface-expressed M1 protein and alter the immunoglobulin binding properties. Res. Microbiol. 149, 539-548

11. Kansal, R. G., Nizet, V., Jeng, A., Chuang, W. J., and Kotb, M. (2003) Selective modulation of superantigen-induced responses by streptococcal cysteine protease. J. Infect. Dis. 187, 398-407

12. Aziz, R. K., Pabst, M. J., Jeng, A., Kansal, R., Low, D. E., Nizet, V., and Kotb, M. (2004) Invasive M1T1 group A Streptococcus undergoes a phase-shift in vivo to prevent proteolytic degradation of multiple virulence factors by SpeB. Mol. Microbiol. 51, 123-134

13. Rezcallah, M. S., Boyle, M. D., and Sledjeski, D. D. (2004) Mouse skin passage of Streptococcus pyogenes results in increased streptokinase expression and activity. Microbiology 150, 365-371

14. McKay, F. C., McArthur, J. D., Sanderson-Smith, M. L., Gardam, S., Currie, B. J., Sriprakash, K. S., Fagan, P. K., Towers, R. J., Batzloff, M. R., Chhatwal, G. S., et al. (2004) Plasminogen binding by group A streptococcal isolates from a region of hyperendemicity for streptococcal skin infection and a high incidence of invasive infection. Infect. Immun. 72, 364-370

15. Sun, H., Ringdahl, U., Homeister, J. W., Fay, W. P., Engleberg, N. C., Yang, A. Y., Rozek, L. S., Wang, X., Sjöbring, U., and Ginsburg, D. (2004) Plasminogen is a critical host pathogenicity factor for group A streptococcal infection. Science 305, 12831286

16. Walker, M. J., McArthur, J. D., McKay, F., and Ranson, M. (2005) Is plasminogen deployed as a Streptococcus pyogenes virulence factor? Trends. Microbiol. 13, 308-313

17. Kazmi, S. U., Kansal, R., Aziz, R. K., Hooshdaran, M., NorrbyTeglund, A., Low, D. E., Halim, A. B., and Kotb, M. (2001) Reciprocal, temporal expression of SpeA and SpeB by invasive M1T1 group A streptococcal isolates in vivo. Infect. Immun. 69, $4988-4995$

18. Schrager, H. M., Rheinwald, J. G., and Wessels, M. R. (1996) Hyaluronic acid capsule and the role of streptococcal entry into keratinocytes in invasive skin infection. J. Clin. Invest. 98, 19541958

19. Hytönen, J., Haataja, S., Gerlach, D., Podbielski, A., and Finne, J. (2001) The SpeB virulence factor of Streptococcus pyogenes, a multifunctional secreted and cell surface molecule with strepadhesin, laminin-binding and cysteine protease activity. Mol. Microbiol. 39, 512-519

20. Ashbaugh, C. D., Warren, H. B., Carey, V. J., and Wessels, M. R. (1998) Molecular analysis of the role of the group A streptococcal cysteine protease, hyaluronic acid capsule, and $\mathrm{M}$ protein in a murine model of human invasive soft-tissue infection. J. Clin. Invest. 102, 550-560

21. Wang, H., Lottenberg, R., and Boyle, M. D. (1994) Analysis of plasmin(ogen) acquisition by clinical isolates of group A streptococci incubated in human plasma. J. Infect. Dis. 169, 143-149

22. Cole, J. N., Ramirez, R. D., Currie, B. J., Cordwell, S. J., Djordjevic, S. P., and Walker, M. J. (2005) Surface analyses and immune reactivities of major cell wall-associated proteins of group A Streptococcus. Infect. Immun. 73, 3137-3146

23. Gillen, C. M., Towers, R. J., McMillan, D. J., Delvecchio, A., Sriprakash, K. S., Currie, B., Kreikemeyer, B., Chhatwal, G. S., and Walker, M. J. (2002) Immunological response mounted by Aboriginal Australians living in the Northern Territory of Australia against Streptococcus pyogenes serum opacity factor. Microbiology 148, 169-178

24. McMillan, D. J., Davies, M. R., Good, M. F., and Sriprakash, K. S. (2004) Immune response to superoxide dismutase in group A streptococcal infection. FEMS Immunol. Med. Microbiol. 40, 249 256

25. Laemmli, U. K. (1970) Cleavage of structural proteins during the assembly of the head of bacteriophage T4. Nature $\mathbf{2 2 7}$, $680-685$

26. Burnette, W. N. (1981) Western blotting: Electrophoretic transfer of proteins from sodium dodecyl sulfate-polyacrylamide gels to unmodified nitrocellulose and radiographic detection with antibody and radioiodinated protein A. Anal. Biochem. 112 195-203

27. Sun, H., Ringdahl, U., Homeister, J. W., Fay, W. P., Engleberg, N. C., Yang, A. Y., Rozek, L. S., Wang, X., Sjöbring, U., and Ginsburg, D. (2004) Plasminogen is a critical host pathogenicity factor for group A streptococcal infection. Science 305, 12831286

28. Rosch, J., and Caparon, M. (2004) A microdomain for protein secretion in Gram-positive bacteria. Science 304, 1513-1515

29. Pancholi, V., and Fischetti, V. A. (1998) $\alpha$-Enolase, a novel strong plasmin(ogen) binding protein on the surface of pathogenic streptococci. J. Biol. Chem. 273, 14503-14515

30. Pancholi, V., and Fischetti, V. (1992) A major surface protein on group A streptococci is a glyceraldehyde-3-phosphatedehydrogenase with multiple binding activity. J. Exp. Med. 176, 415-426

31. Wang, H., Lottenberg, R., and Boyle, M. D. (1995) A role for fibrinogen in the streptokinase-dependent acquisition of plas$\min ($ ogen) by group A streptococci. J. Infect. Dis. 171, 85-92

32. Werb, Z. (1997) ECM and cell surface proteolysis: regulating cellular ecology. Cell 91, 439-442

33. Berge, A., and Sjöbring, U. (1993) PAM, a novel plasminogenbinding protein from Streptococcus pyogenes. J. Biol. Chem. 268, 25417-25424

34. Lottenberg, R., Broder, C. C., Boyle, M. D., Kain, S. J., Schroeder, B. L., and Curtiss, R., 3rd (1992) Cloning, sequence analysis, and expression in Escherichia coli of a streptococcal plasmin receptor. J. Bacteriol. 174, 5204-5210

35. Wang, H., Lottenberg, R., and Boyle, M. D. (1995) Analysis of the interaction of group A streptococci with fibrinogen, streptokinase and plasminogen. Microb. Pathog. 18, 153-166

36. Boyle, M. D., and Lottenberg, R. (1997) Plasminogen activation by invasive human pathogens. Thromb. Haemost. 77, 1-10

37. Herwald, H., Cramer, H., Morgelin, M., Russell, W., Sollenberg, U., Norrby-Teglund, A., Flodgaard, H., Lindbom, L., and Björck, L. (2004) M protein, a classical bacterial virulence determinant, forms complexes with fibrinogen that induce vascular leakage. Cell 116, 367-379

Received for publication January 20, 2006. Accepted for publication March 31, 2006. 


\title{
Trigger for group A streptococcal M1T1 invasive disease
}

\author{
Jason N. Cole,* Jason D. McArthur,* Fiona C. McKay,* Martina L. Sanderson-Smith,* \\ Amanda J. Cork, ${ }^{*}$ Marie Ranson, ${ }^{*}$ Manfred Rohde, ${ }^{\dagger}$ Andreas Itzek, ${ }^{\dagger}$ Hongmin Sun, ${ }^{\ddagger}$ \\ David Ginsburg, ${ }^{+,},, \|, \#$ Malak Kotb, ${ }^{* *}$ Victor Nizet, ${ }^{\dagger+}$ G. S. Chhatwal, ${ }^{\dagger}$ \\ and Mark J. Walker*,1 \\ *School of Biological Sciences, University of Wollongong, Wollongong, New South Wales, Australia; \\ ${ }^{\dagger}$ Department of Microbial Pathogenesis and Vaccine Development, German National Centre \\ for Biotechnology, Braunschweig, Germany; ${ }^{\dagger}$ Life Sciences Institute, University of Michigan, \\ Ann Arbor, Michigan, USA; ${ }^{{ }^{2}}$ Howard Hughes Medical Institute, ${ }^{\|}$Department of Internal Medicine \\ and "Department of Human Genetics, University of Michigan, Ann Arbor, Michigan, USA; \\ **Department of Molecular Science, University of Tennessee, Memphis, Tennessee, USA; and \\ ${ }^{\dagger}$ Department of Pediatrics, UCSD, La Jolla, California, USA
}

To read the full text of this article, go to http://www.fasebj.org/cgi/doi/10.1096/fj.05-5804fje

\section{SPECIFIC AIMS}

The globally disseminated M1T1 clone of Streptococcus pyogenes (group A Streptococcus; GAS) causes a number of highly invasive human diseases, including necrotizing fasciitis and streptococcal toxic shock-like syndrome. Although the transition from local to systemic infection occurs by an unknown mechanism, invasive M1T1 clinical isolates express significantly less cysteine protease SpeB than M1T1 isolates from localized infections. Subversion of the host plasminogen activation system by GAS may also play a key role in systemic disease initiation. In this study, we utilize the representative wild-type (WT) clonal M1T1 isolate 5448 and the isogenic mutant $\Delta s p e B$ to investigate the interaction between SpeB and human plasminogen in GAS invasive disease. The specific aims were: 1 ) to characterize capsule expression, SpeB protease activity, plasminogen-binding capacity, and accumulation of surface-bound plasmin activity in WT and $\Delta s p e B$ strains; 2) to examine the virulence of $\mathrm{WT}$ and $\Delta s p e B$ strains in a humanized plasminogen transgenic mouse model of subcutaneous (s.c.) infection; and 3) to analyze the SpeB activity of WT colonies isolated from the skin lesions and blood of infected plasminogen-humanized mice.

\section{PRINGIPAL FINDINGS}

\section{Characterization of M1T1 5448 and $\Delta s p e B$}

The WT and $\Delta s p e B$ mutant expressed identical amounts of hyaluronic acid capsule (Fig. 1a), excluding a pleiotropic mutation that confounded earlier studies of SpeB in GAS pathogenesis. SpeB protease activity was undetectable in the $\Delta$ speB mutant
$(P \leq 0.05$; Fig. $1 b)$, and SpeB zymogen was identified only at the ExPortal microdomain of the WT strain (Fig. 1c). Nonetheless, equivalent amounts of human plasminogen were bound by the WT and $\Delta s p e B$ mutant (Fig. $1 d$ ), suggesting that SpeB does not affect the capacity of M1T1 GAS to bind human plasminogen. Compared with the WT strain, $\Delta s p e B$ accumulated 75-fold higher levels of plasmin activity on the bacterial surface following incubation in human plasma ( $P \leq 0.05$; Fig. $1 e)$. These data suggest that SpeB may interfere with the accumulation of plasmin on the GAS cell surface.

\section{Infection of humanized plasminogen transgenic mice}

Compared with nontransgenic control mice $\left(\mathrm{Tg}^{-}\right)$, the virulence of the WT strain was significantly enhanced in humanized plasminogen transgenic mice $\left(\mathrm{Tg}^{+}\right) \quad(P \leq 0.05 ; 10 \%$ vs. $80 \%$ mortality) (Fig. $2 \boldsymbol{a}$ ), indicating that human plasminogen is essential for M1T1 virulence. In comparison to the WT GAS parent strain, the $\Delta s p e B$ mutant was attenuated for virulence in the $\mathrm{Tg}^{+}$mice $(P \leq 0.05 ; 80 \%$ vs. $20 \%$ mortality) (Fig. $2 a, b)$ and displayed significantly fewer bacterial counts in the blood $(P \leq 0.05$; Fig. $2 c)$. These data support a role for SpeB in GAS survival at the site of local skin infection.

\footnotetext{
${ }^{1}$ Correspondence: School of Biological Sciences, University of Wollongong, Wollongong, NSW 2522, Australia. E-mail: mwalker@uow.edu.au

doi: 10.1096/fj.06-5804fje
} 
a
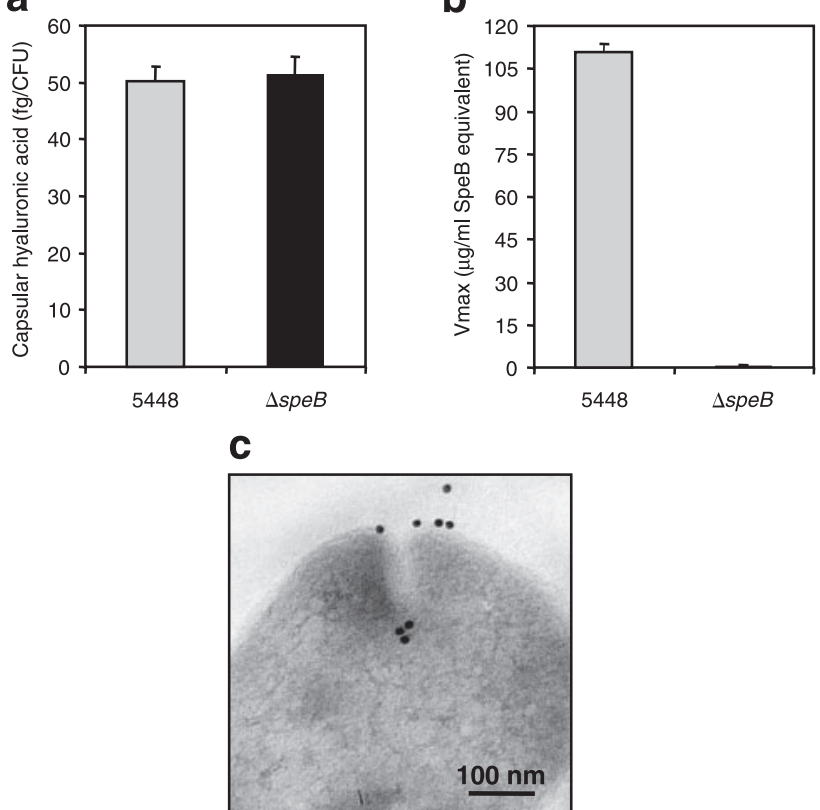

d

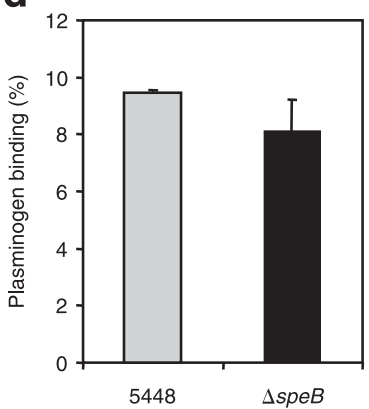

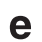

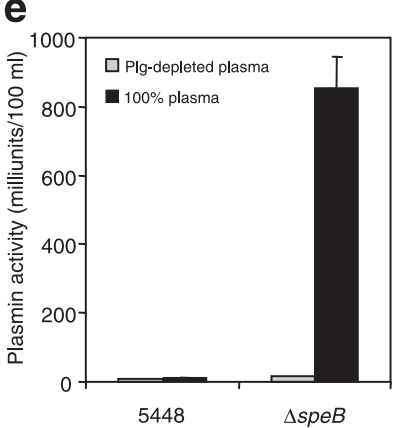

Figure 1. In vitro characterization of M1T1 S. pyogenes strain 5448 (WT) and the isogenic $\Delta$ speB mutant. a) 5448 and the $\Delta s p e B$ mutant express equivalent amounts of capsular hyaluronic acid (mean \pm SD). b) Secreted SpeB protease activity (mean $\pm \mathrm{SD}$ ) is abolished in the $\Delta$ speB mutant compared with 5448. c) Electron microscopic analyses detected SpeB zymogen at the ExPortal microdomain of 5448, but not $\Delta$ speB (data not shown). d) Equivalent amounts of purified human ${ }^{125}$ I-labeled plasminogen $($ mean \pm SD) are bound by 5448 and $\Delta s p e B$. e) The $\Delta s p e B$ mutant cell surface accrues a 75 -fold higher concentration of human plasma activity (mean \pm SD) compared with strain 5448 .

\section{SpeB activity of 5448 in vivo isolates}

The WT strain used in the initial challenge uniformly expressed high levels of SpeB protease activity $(100 \%$ of inoculum colonies was SpeB-positive; $n=100)$. WT bacteria recovered from the lesions of four $\mathrm{Tg}^{+}$mice expressed a mixed SpeB phenotype $(74 \%, 66 \%, 63 \%$ and $31 \%$ of lesion colonies examined were SpeBnegative). However, WT bacteria recovered from the blood unvaryingly demonstrated very low SpeB activity $(100 \%$ of blood colonies examined were SpeB-negative) (Fig. 2d). This finding is consistent with the epidemiologic observation that M1T1 clinical isolates

a

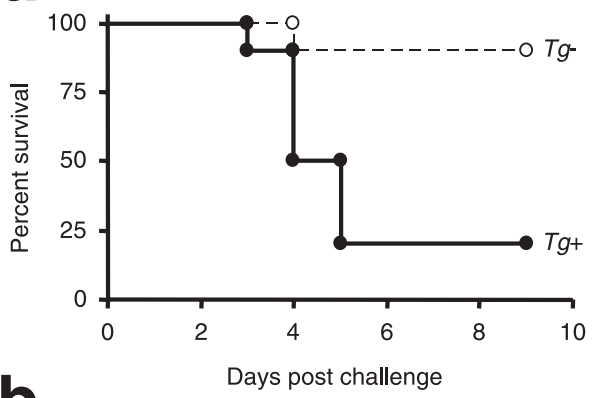

b

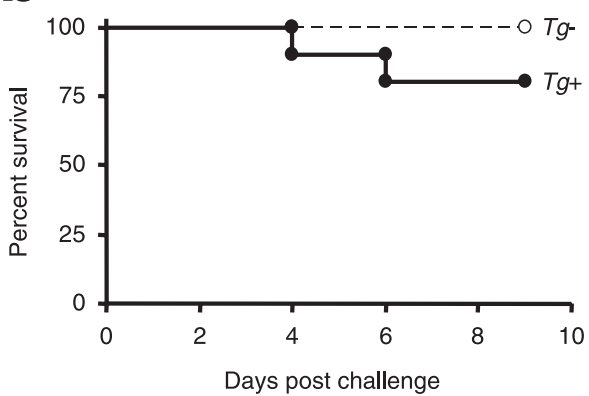

C
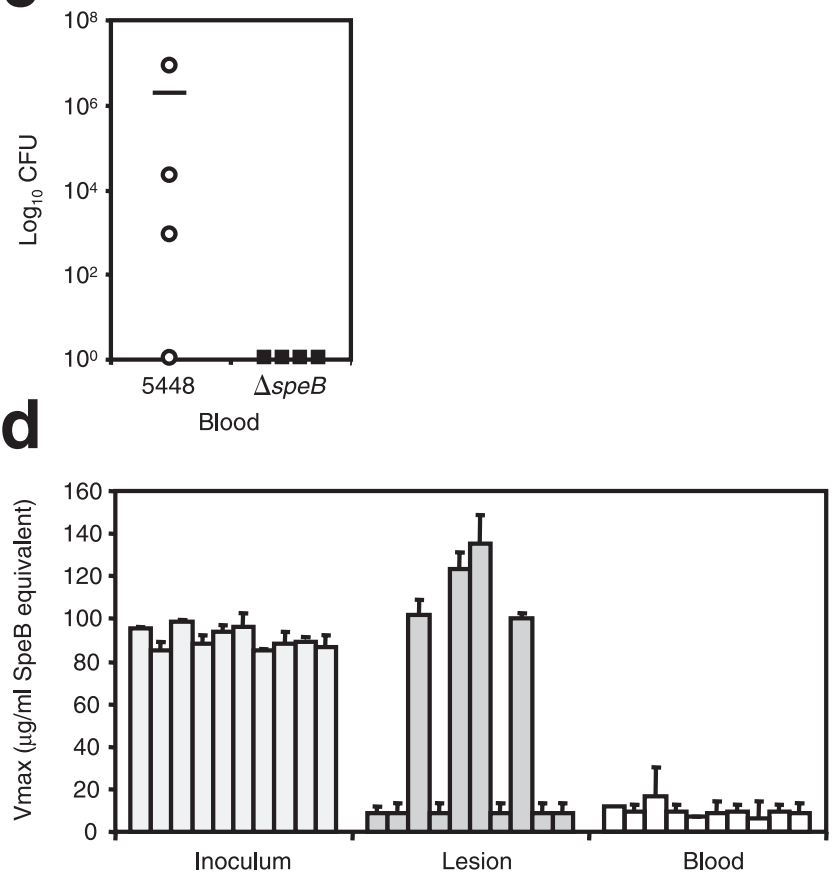

Figure 2. Survival curves, bacterial counts and SpeB expression phenotype following s.c. infection of humanized plasminogen transgenic mice $\left(T g^{+}\right)$and nontransgenic control mice $\left(\mathrm{Tg}^{-}\right)$with $S$. pyogenes strain 5448 (WT) or the isogenic $\Delta$ speB mutant. a) The virulence of 5448 is increased in transgenic mice compared with the nontransgenic control. $b$ ) The virulence of the $\Delta s p e B$ mutant was attenuated in both transgenic and control mice. c) Bacterial counts in the bloodstream of $\mathrm{Tg}^{+}$mice $72 \mathrm{~h}$ postinfection are significantly higher for 5448 compared with $\Delta s p e B$. d) Compared with the SpeB activity of 5448 colonies isolated from the inoculum, the $5448 \mathrm{Tg}^{+}$lesion isolates exhibit a varied SpeB-expression phenotype, while the $5448 \mathrm{Tg}^{+}$blood isolates have significantly reduced $\mathrm{SpeB}$ activity. 


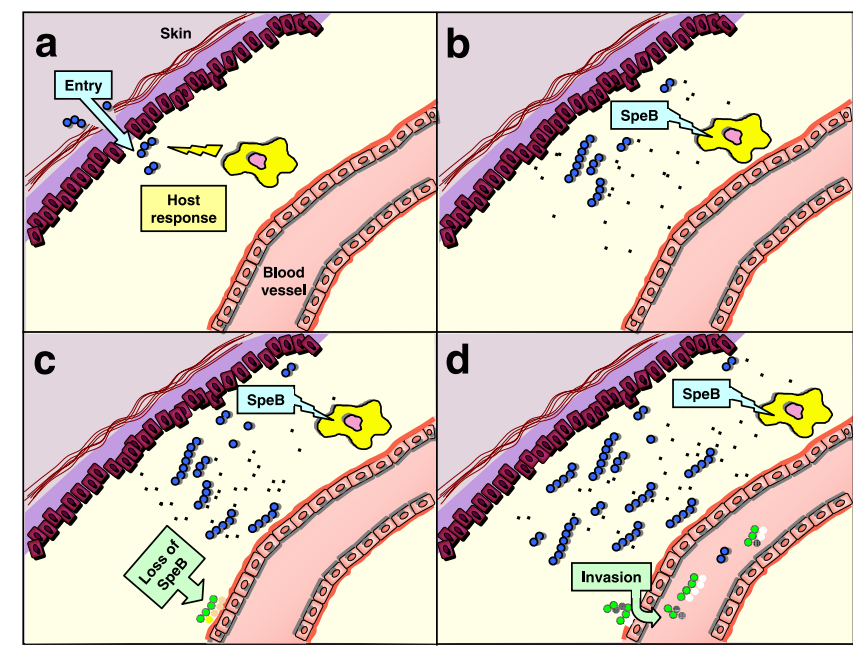

Figure 3. Proposed model for group A streptococcal systemic disease initiation. a) S. pyogenes M1T1 (blue) gain entry through the skin and a host innate immune response is initiated. $b$ ) During the initial stages of infection, $S$. pyogenes M1T1 express SpeB to combat the host response. c) Loss of SpeB activity in a subpopulation of group A streptococci (green) leads to the accumulation of surface plasmin activity. d) Transition of $S$. pyogenes M1T1 is facilitated by surface plasmin activity, resulting in systemic infection.

from human invasive disease express reduced levels of SpeB.

\section{CONCLUSIONS AND SIGNIFICANGE}

The mechanism utilized by GAS to switch from localized to systemic infection is presently unknown. In this study, we have shown that the absence of cysteine protease SpeB activity is required for the accumulation of plasmin activity on the cell surface of M1T1 GAS. In a humanized plasminogen transgenic mouse model of s.c. infection, the loss of SpeB activity at the site of infection triggers the systemic dissemination of M1T1 GAS in vivo. These data support the proposal that human plasminogen plays a critical role in the initiation of GAS invasive disease.

Our accumulated data suggest that SpeB, while contributing to localized infection, simultaneously mitigates the potential interaction of M1T1 GAS with the human plasminogen activation system. The ablation of SpeB expression in WT M1T1 GAS in the blood of plasminogen-humanized $\mathrm{Tg}^{+}$mice indicates that the loss of SpeB activity in a subpopulation of bacteria occurs at the site of infection, allowing accumulation of surface plasmin activity. This subpopulation thus gain enhanced invasive propensity, favoring transition of GAS from the site of infection to the blood. In our model, vascular leakage induced by M protein complexing with fibrinogen to activate heparin binding protein release from neutrophils, may provide a source of plasminogen at the site of infection (Fig. 3).

The expression of the streptococcal cysteine protease SpeB and human invasive disease severity are inversely related in M1T1 clonal isolates. The model we propose accounts for this previously perplexing clinical observation and describes a mechanism by which systemic disease initiation occurs. The elucidation of the mechanism by which GAS causes human invasive disease will inform future treatment and prevention strategies. F] 